\title{
LEITUNG
}

\section{Führen ohne Weisungsbefugnis}

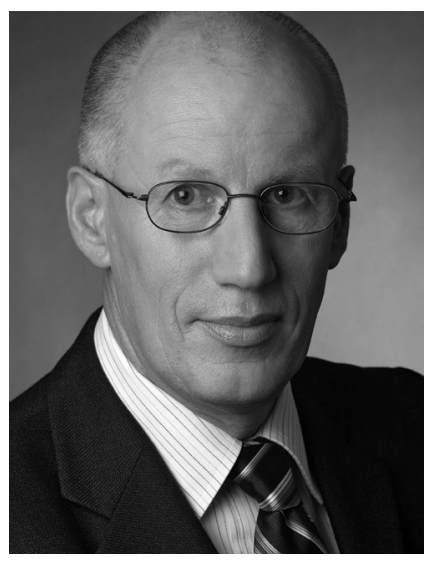

VON JOHANN SCHOLTEN Johann Scholten ist einer der drei Geschäftsführer der WSFBBeratergruppe Wiesbaden, die Unternehmen bei Veränderungsprozessen begleitet und deren Mitarbeiter trainiert. Zudem bildet die Gruppe Organisationsberater aus.

www.wsfb.de

\author{
Je netzwerkartiger die Arbeitsstrukturen und \\ Organisationsbeziehungen in den Unternehmen \\ werden, umso häufiger müssen Mitarbeiter auch \\ Personen führen, deren Vorgesetzte sie nicht sind. \\ Dieses sogenannte laterale Führen bereitet oft \\ allen Beteiligten nicht wenige Schwierigkeiten.
}

Die Organisationsstrukturen auch sozialer Unternehmen haben sich in den vergangenen Jahrzehnten stark gewandelt. Dasselbe gilt für die Art, wie sie ihre Leistungen erbringen. In den tayloristisch organisierten Betrieben und bürokratischen Verwaltungen der Vergangenheit standen die Bereiche weitgehend unverknüpft, gleich Säulen nebeneinander. Und jeder Bereich hat sein klar definiertes Aufgabenfeld. Ebenso war dies bezogen auf die Mitarbeitenden. Auch von ihnen hatte jeder klar umrissene Aufgaben, die entweder in seiner Stellenbeschreibung definiert oder ihm von seinem Vorgesetzten übertragen worden waren.

Anders ist dies heute zumindest in vielen Organisationen und Betrieben: Sie sind weitgehend netzwerkartig strukturiert. Und die Bereichsgrenzen sowie Hierarchiestufen spielen in ihrer Alltagsarbeit eine immer geringere Rolle - unter anderem, weil die Leistungen zunehmend in bereichs- und oft sogar unternehmensübergreifenden Arbeitsteams erbracht werden.

Damit stößt das klassische Führen an seine Grenzen, das weitgehend auf der qua Position verliehenen disziplinarischen Macht und Weisungsbefugnis beruht. An Bedeutung gewinnt hingegen das sogenannte laterale Führen, das auf Vertrauen und Verständigung beruht und danach strebt, durch das Schaffen eines gemeinsamen Denkrahmens die unterschiedlichen Interessen der Beteiligten soweit möglich tragfähig zu verbinden.
Diese Art von Führung muss sich, da die disziplinarische Weisungsbefugnis als Machtquelle entfällt, auf andere Machtquellen stützen - zum Beispiel eine hohe persönliche Autorität und Integrität. Oder auf ein ausgewiesenes Expertentum. Oder auf ein gezieltes Networking, das die eigene informelle Machtbasis stärkt.

Dabei stellt der Begriff »Laterale Führung «, zumindest ausgehend vom klassischen Führungsverständnis, einen Widerspruch in sich selbst dar. Denn ihm zufolge ist Führung untrennbar mit einer hierarchischen Weisungsbefugnis und Unterordnung verbunden. Trotzdem ist laterale Führung zunehmend eine typische Erfordernis der heutigen Organisationswelt, die durch bereichsübergreifende Kooperationen, Vernetzungen, flache Hierarchien sowie Teamund Projektarbeit gekennzeichnet ist.

Wichtig ist dabei festzuhalten: Lateral führen bedeutet mehr als koordinieren. Denn während Koordination primär auf ein Aufeinanderabstimmen zum Beispiel von Interessen, Aufgaben oder Tätigkeiten abzielt, beinhaltet Führung auch ein Einwirken auf andere Personen oder Organisationen, damit sie in eine gewünschte Richtung handeln.

Zentrales Ziel von lateraler Führung ist also nicht das Aushandeln oder Vereinbaren tragfähiger Kompromisse, sondern das Erreichen der eigenen oder übergeordneten Ziele (zum Beispiel des Unternehmens), wozu wiederum der Kompromiss ein Instrument sein kann. 
Entsprechend schwierig ist laterale Führung in der Praxis, da die an diesem Prozess beteiligten Personen zum Beispiel aufgrund ihrer unterschiedlichen Funktion und Position in der Organisation häufig divergierende Auffassungen und Interessen haben.

Dessen ungeachtet gewinnt aufgrund der heutigen Organisation von Unternehmen das Thema laterale Führung an Bedeutung. Einige Beispiele, wann laterale Führung häufig gefragt ist, seien genannt:

- Ein Projektmanager oder Teamleiter erhält von seinem Vorgesetzten zwar ein Mandat, die Kooperation in Gang zu setzen und zu halten, er hat aber nur schwache Mittel, die Kooperanden »an die Leine « zu nehmen.

- Ein Bereich muss mit Nachbarbereichen entlang der Prozesskette kooperieren, der Bereichsleiter kann bei Meinungsverschiedenheiten aber nicht auf übergeordnete Instanzen als »Schlichter « zurückgreifen - zum Beispiel aufgrund von Zeitmangel, fehlender Praxisnähe der Instanzen oder mangelnder Konfliktschlichtungskapazität.

- Ein Wohlfahrtsverband muss sich mit mehreren juristisch autonomen Mitgliedsorganisationen darüber verständigen, wer im Rahmen eines gemeinsamen Großprojekts gewisse Leistungen für das geplante Vorhaben erbringt.

Von hoher Bedeutung ist laterale Führung auch beim Treffen und Herbeiführen von Entscheidungen in Kollegialgremien - wie zum Beispiel Vorständen oder Beiräten - sowie in bereichs- und unternehmensübergreifenden Veränderungsprozessen, beispielsweise bei Fusionen.

Da beim lateralen Führen das Erteilen von Anweisungen entfällt, bedarf es anderer, eher informeller Instrumente der Führung. Diese lassen sich vier Komplexen zuordnen.

- Denkgebäude öffnen, um Verständigung herzustellen: Auffassungen (= mentale Modelle) verändern, damit Verständigung entsteht; individuelle mentale Modelle erweitern, um kollektive mentale Modelle zu kreieren; das Lernen 1. Ordnung (Verhalten) ergänzen um ein Lernen 2. Ordnung (mentale Modelle, Werte); sondieren der individuellen Interessen, um Ziele, Herausforderungen, professionelle Werte zu klären; sondieren der individuellen Auffassungen, um Überzeugungen, Arbeitsschemata, Entscheidungsverfahren zu klären

- Kommunikation verbessern: Verständigungsbrücken schaffen (Partner sollen nachvollziehen können, was der andere meint); tatsächliche Interaktion, Dialoge entstehen lassen (die einen reagieren auf die Äußerungen der anderen in ihrer Meinungsbildung)

- Vertrauen erzeugen und erhalten: Vertrauen als Tauschgeschäft (Wer vertraut, dem wird vertraut. Einer beginnt und wartet die Reaktion des Anderen ab, um dann seinerseits wieder zu reagieren.); Grundlagen von persönlichem Vertrauen herausarbeiten lassen; Zwänge offen legen; Regeln der Kommunikation und Kooperation erarbeiten, einführen und kontrollieren; Vertrauen langsam wachsen lassen (Felder identifizieren, wo das Risiko nicht so groß ist.)

- Macht arrangieren: Macht erwächst aus dem Beherrschen von Unsicherheitszonen, die es zu reduzieren gilt (zum Beispiel aufgrund von Fachwissen, Markt-, Umweltbeziehungen, Kommunikation, formalen Bedingungen, Charisma, Heldentaten, Sympathie, Freundlichkeit, Zukunftseinschätzungen); Macht ist eine Austauschbeziehung, getauscht werden Handlungsmöglichkeiten (= Fähigkeit, für andere wichtige Probleme zu lösen). Dafür ist ein "Markt « $\mathrm{zu}$ schaffen (neue Austauschbeziehungen entstehen lassen und so das Machtgefüge verändern); Coaching, Beratung in Anspruch nehmen, um eine Außenperspektive einzuführen und durch angeleitete Reflexion Blockaden zu überwinden; ein gemeinsames Ziel, eine gemeinsame Vision kreieren; Erhöhung der Rationalität durch Weitergabe von Wissen und Informationen

\section{Resümee: Oberste Aufgabe ist die Zielerreichung}

Die vier Steuerungsmechanismen »Kommunikation verbessern«, »Verständigung erreichen «, »Machtgefüge arrangieren « und »Vertrauen aufbauen $«$ greifen ineinander und lassen sich im Unternehmens- und Führungsalltag schwer trennen. Sie laufen immer gleichzeitig und können sich gegenseitig stützen, behindern oder ersetzen.

Laterale Führung folgt besser einer Logik der Kontingenz statt Konsequenz. Das heißt: Laterale Führungskräfte halten sich für die Auffassungen und Interessen der anderen offen. Laterale Führung lässt zudem in der Entscheidungsfindungsphase offen, welches Entscheiden und Handeln das richtige oder beste ist (»Es könnte so gehen, aber auch anders - jedoch nicht beliebig! «).

Denn wichtig ist es nicht, den (scheinbar) »besten « Weg durchzusetzen, sondern das übergeordnete Ziel zu erreichen. Und hierfür gibt es zumeist mehrere Wege.

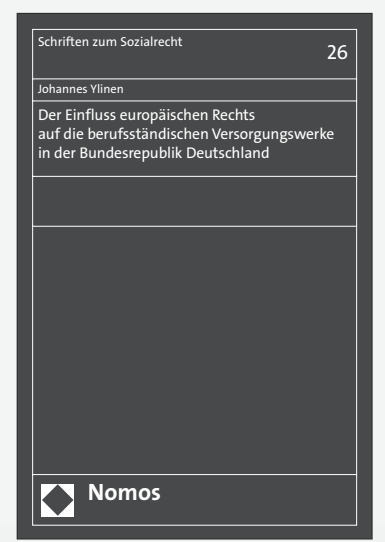

Der Einfluss europäischen

Rechts auf die berufsständischen Versorgungswerke in der Bundesrepublik Deutschland

Von Johannes Ylinen

2013, 206 S., brosch., 46,-€, ISBN 978-3-8487-0162-9

(Schriften zum Sozialrecht, Bd. 26)

Die Arbeit untersucht die Auswirkungen des Rechts der EU auf ein Sondergebiet des deutschen Kammerrechts, das Recht der Versorgungswerke der freien Berufe in der Bundesrepublik.

www.nomos-shop.de/20316

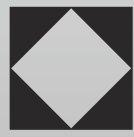

Nomos 